\title{
XV. FURTHER OBSERVATIONS ON THE TRANSMISSION OF PLAGUE BY FLEAS, WITH SPECIAL REFERENCE TO THE FATE OF THE PLAGUE BACILLUS IN THE BODY OF THE RAT FLEA (P. CHEOPIS).
}

We have previously (vol. vi. p. 446) shown that plague may be transferred from rat to rat by the transference of fleas from a septicaemic to a healthy animal. We propose in the present communication to deal with the following questions:-

I. The fate of the plague bacillus in the body of the rat flea.

II. Whether a single rat flea can transmit the infection.

III. Whether both male and female rat fleas can transmit the infection.

IV. Whether other species of fleas can transmit the infection.

V. The mechanism by which the flea infects a healthy animal.

I. Fate of the Plague Bacilli in the Body of the Rat Flea ( $P$. cheopis).

\section{Capacity of Flea's Stomach.}

We have already (vol. vI. p. 486) described the physiological anatomy of the mouth parts and of the alimentary canal of the Indian rat flea, Pulex cheopis. We have described how the blood is sucked up from the wound made by the pricking apparatus of the insect through the mouth into the pharynx, how it is then passed down the pharynx and oesophagus by a successive wave of contraction of the muscles from before backwards, and how from the oesophagus it passes into the stomach, at the anterior end of which is a valvular arrangement called "the gizzard." The stomach is a pear-shaped organ occupying a considerable part of the abdomen of the insect. That it is capable of containing a considerable amount of blood is apparent from the 
observation that after a flea has had a meal it is seen that nearly the whole of the abdomen is filled with a bright red mass.

We have made an endeavour to measure as accurately as possible the average capacity of the stomach when filled with blood. Two methods were employed, namely, (a) direct measurement, (b) indirect estimation.

(a) Direct measurement of Flea's Stomach.

Healthy fleas, taken from Bombay rats, were starved for 12 hours, and at the end of that time fed on healthy animals. The stomach was then dissected out whole and floated in normal salt solution, adherent tracheae and the fat body being removed. Under these conditions the organ assumes a regular ellipsoidal form and direct measurement can be employed. The microscope was adjusted to a magnification of exactly 100 diameters and the image of the stomach floating in salt solution was projected on paper. The outline was traced, and then measured, the measurements being put through the "Simpson's first rule." The method was controlled by taking globules of mercury of known volume, and putting them through the same process. It was found to be accurate to $1 / 10$ of a cubic millimetre but not to one hundredth. Working in this way we have obtained the following results:-

(a) Volume of very full and distended stomach from large flea, $0.7-0.75$ c.mm.

(b) Tolume of full stomach of average sized flea, $0.38-0.48$ c.mm.

It is to be noted that this method gives the total volume of the stomach, including the stomach walls. It is an outside estimate.

(b) Indirect estimation of capacity of Flea's Stomach.

In this method a comparison was made between the tint of a solution of the contents of several stomachs and that of standard solutions of carbonic oxide haemoglobin.

A series of 10 standards, namely 1 to 10 p.c., of dilutions of rats' blood, through which carbonic oxide had been passed, was prepared. These were put up in calibrated capillary tubes, and arranged side by side on a card. Five fleas, which had just fed, were then dissected and their stomach contents dissolved in a known quantity of distilled water, and $\mathrm{CO}$ passed through. The resulting fluid was introduced into a capillary tube of the same calibre as the standards, and a comparison made with the standards. Having ascertained the standard tube to which it corresponded in tint, an easy calculation determined the volume of blood in the stomachs. 
The following results were obtained :-

(a) Large distended stomach - average of 5 stomachs $=0.3 \mathrm{c.mm}$.

(b) Large distended stomach - average of 5 stomachs $=0.25 \mathrm{c.mm}$.

(c) Small half filled stomach - average of 5 stomachs $=0.12 \mathrm{c.mm}$.

It will be noted that these estimations are considerably lower than those obtained by direct mensuration. This result was anticipated, as in the direct method the walls of the stomach are included, and the result is therefore certainly too high. The haemoglobin method, on the other hand, gives too low an estimate, as it is based on a comparison with whole fresh blood in the standards, while the stomach of the flea contains, mixed with the fresh blood of the last meal, a large proportion of residuum of darkly coloured semi-digested material of the previous meals. It is also impossible to arrest the process of digestion immediately after feeding. For our present purpose, therefore, we shall not be greatly in error if we consider the average volume of blood capable of being accommodated in the stomach of a rat flea at each meal to be about $0.5 \mathrm{c} . \mathrm{mm}$.

\section{Number of Plague Bacilli taken into the Stomach.}

In our first report (vol. vi. p. 519) we have shown that the blood of a plague-infected rat may contain an enormous number of plague bacilli, even as many as 100,000,000 per c.c., having been found before death. If, therefore, a rat flea imbibed the blood of such a rat, it would receive into its stomach, allowing the capacity of this organ to be $0.5 \mathrm{c} . \mathrm{mm} ., 5000$ plague germs. It is further evident that a flea which imbibed the blood of a rat containing 10,000 or more plague germs per c.c. would take some bacilli into its stomach. From the table published in vol. vr. p. 521 it will be seen that about two-thirds of plague rats, either before death or immediately after death, contain more than this number of germs in their blood, so that fleas would have ample opportunity of taking bacilli into their stomachs.

\section{Summary.}

1. The average capacity of a rat flea's stomach has been approxi- . mately estimated to be $0.5 \mathrm{c.mm}$.

2. A rat flea imbibing blood from a plague-infected rat might receive as many as 5000 germs into its stomach.

3. Fleas feeding on a large proportion of plague-infected rats just before death would imbibe some plague bacilli. 


\section{Multiplication of Plague Bacilli in the Stomach of the Rat Flea'.}

Several observers have previously noted what they consider to be a multiplication of plague bacilli in the stomachs of fleas which had fed on plague-infected rats. The evidence of multiplication having taken place was in all instances the observation that the contents of the flea's stomach, on being squeezed out on a slide and stained, contained abundant bipolar stained bacilli which stained well and showed no involution forms. No account was taken of the interval between the ingestion of the septicaemic blood and the examination of the stomach contents. It is obvious that such evidence of 'multiplication is very incomplete, inasmuch as we know that soon after a flea has filled its stomach, absorption begins and the blood in the stomach is greatly reduced in volume, finally becoming a thick tarry mass.

The number of bacilli seen in a preparation of the stomach contents at this time will appear to be greatly in excess of the number which is to be found in any preparation of rats' blood, so that a false impression that multiplication has taken place might be conveyed.

As time goes on, the tarry mass in the stomach passes on into the rectum, this process being greatly accelerated by the fresh blood which the flea takes into its alimentary canal. We have already drawn attention to the habit which the flea, in common with other bloodsucking insects, possesses, of squirting bright red blood from the anus during the operation of sucking. It is evident that the whole of the alimentary canal is being subjected to a regular process of washing out. If then fleas, taken from septicaemic plague rats, are fed on healthy animals, a fresh animal being used each day, so that there is no chance of the fleas taking a second supply of bacilli into their stomachs, and if

1 When we talk of "plague bacilli" in the stomach of a flea, we mean that there was present an organism which was microscopically identical with the plague bacillns. The evidence that this organism is really the plague bacillus is based mainly on the observation that no such organism has ever been found by us in the stomach contents of fleas taken from healthy rats. It is only when fleas have fed on plague-infected rats containing plague bacilli in their blood that a bipolar stained bacillus is found in their stomach contents. We have also the evidence that the faeces of fleas taken from plague. infected rats gives plague to animals and that the fleas themselves can transmit the disease to other animals.

We have already described (vol. vi. p. 492) the method by means of which the abdominal organs of a flea are dissected out. After these organs have been separated from one another the stomach is opened with a sharp needle and its contents, mixed with a little salt solution, smeared over the slide. 


\section{Reports on Plague Investigations in India}

at the end of several days the stomach contents are found to be crowded with plague bacilli, we can only conclude that multiplication of the original bacilli has taken place. Had multiplication not taken place the successive diluting and sweeping out processes which had been going on would certainly have cleared out the stomach of most of the bacilli originally taken in.

We have accordingly made a large series of observations, which had as their object the determination of the presence or absence of abundant plague bacilli in the stomach contents of fleas at varying intervals after they had imbibed septicaemic blood, the fleas being fed in the meantime on the blood of healthy animals.

These observations were made during the months of December to April, which is the epizootic and epidemic plague season in Bombay. The method was as follows. Bombay rats were inoculated with virulent cultures of plague bacilli. When sick they were placed separately in flea-proof cages and a number of fleas, which had been taken from wild Bombay rats, were put in with them. The rats were removed from the cages as soon after death as possible, any fleas which were still on them being caught and examined. For our further observations only those cages were used which had contained rats showing a well-marked septicaemia at death. The same day a fresh healthy rat or guinea-pig was put into the cage and kept in for 24 hours. It was then removed and segregated, some of the fleas which were caught on it being dissected, while the others were returned to the cage. The same process was repeated each day. Thus, on each succeeding day, the fleas were supplied with the blood of a healthy animal so that there could be no question of a fresh infection with plague bacilli. The fleas which were removed from the cage each day were dissected: a smear of the stomach contents was made, stained with thionin-blue and examined microscopically, the presence or absence of abundant plague bacilli being noted.

The results of these examinations have been brought together on the basis of the interval between the probable time at which the fleas imbibed the plague bacilli and the time at which the examination of the stomach contents was made. It is, of course, impossible to determine exactly when the fleas took their last meal containing bacilli, as they were in the same cage with the sick rats for from 12 to 48 hours before the death of the latter. On several occasions we made attempts to fix definitely the hour at which the fleas took the bacilli into their bodies. This was done by taking rats which were evidently very ill with plague, 
confining their movements and allowing fleas to feed on them through a layer of muslin covering the end of a glass tube. It was, however, soon found that a rat sick with plague will not stand handling as it dies almost immediately from heart failure. This attempt, therefore, had to be abandoned, as it was only on the rarest occasions that the fleas were able to obtain blood containing any plague bacilli.

While, then, in these observations we cannot definitely fix the time at which the fleas took the plague bacilli into their stomachs, it is more than probable that the great majority of these, in the stomachs of which bacilli were found in large numbers, had fed on septicaemic blood within twelve hours of the rat's death. For we know that fleas feed frequently when left to their own inclinations, and that they are especially fond of animals which are sick and unable to clean themselves.

\section{TABLE I.}

Results of examination of rat fleas as to presence of abundant plague bacilli in stomach contents, day by day after their being taken from septicaemic rats, the fleas in the meantime being fed on healthy animals. December to April.

\begin{tabular}{|c|c|c|c|c|}
\hline \multicolumn{2}{|c|}{$\begin{array}{l}\text { Interval between } \\
\text { ingestion of } \\
\text { septicaemic blood and } \\
\text { examination of tlea }\end{array}$} & $\begin{array}{l}\text { Number } \\
\text { of fleas } \\
\text { dissected }\end{array}$ & $\begin{array}{c}\text { Number of fleas } \\
\text { found with abundant } \\
\text { plague bacilli } \\
\text { in stomach contents }\end{array}$ & $\begin{array}{l}\text { Percentage of } \\
\text { fleas with abundant } \\
\text { plague bacilli } \\
\text { in stomach contents }\end{array}$ \\
\hline \multicolumn{2}{|c|}{1 day } & 54 & 22 & 47 \\
\hline \multicolumn{2}{|c|}{2 days } & 16 & 6 & 38 \\
\hline 3 & ", & 24 & 9 & 37 \\
\hline 4 & , & 28 & 15 & 53 \\
\hline 5 & , & 37 & 8 & 21 \\
\hline 6 & $n$ & 33 & 5 & 15 \\
\hline 7 & ", & 25 & 3 & 12 \\
\hline 8 & , & 18 & 3 & 16 \\
\hline 9 & , & 13 & 0 & 0 \\
\hline 10 & , & 15 & 1 & 7 \\
\hline 11 & ", & 17 & 2 & 12 \\
\hline 12 & $"$ & 22 & 2 & 9 \\
\hline 13 & ", & 16 & 0 & 0 \\
\hline 14 & " & 13 & 0 & 0 \\
\hline 15 & , & 6 & 0 & 0 \\
\hline 16 & , & 16 & 0 & 0 \\
\hline 17 & , & 14 & 0 & 0 \\
\hline 18 & , & - & - & - \\
\hline 19 & , & 14 & 0 & 0 \\
\hline 20 & ," & 15 & 1 & 7 \\
\hline 21 & $"$ & 19 & 0 & 0 \\
\hline 22 & $"$ & - & - & - \\
\hline 23 & " & 13 & 0 & 0 \\
\hline
\end{tabular}


For our present purpose, therefore, we have taken 12 hours before the death of the rat as the time when the fleas imbibed the bacilli, and as most of the rats died during the night the day on which they were found dead is reckoned as one day after the fleas took the germs into their stomachs, the next day as two days, and so on. Throughout this paper we shall talk of "first day fleas," "second day fleas," etc., and the meaning which is to be given to these expressions is as we have explained.

Table I contains the results of the present observations. From this table it is seen that day by day, up to the 12th day, a considerable percentage of the fleas examined contained abundant plague bacilli in the contents of their stomachs, and that, while all fleas examined on the 13th to 19th days contained no bacilli, a single specimen out of 15 dissected on the 20th day showed abundant germs in its stomach.

\section{Summary.}

Fleas were fed on plague-infected rats until the death of these animals. They were afterwards fed on healthy animals. A number were dissected each day for 23 days. In a certain proportion abundant plague bacilli were found in the stomach contents up to the 12 th day and in one instance on the 20th day. We have good evidence in this observation that multiplication of plague bacilli may take place in the flea's stomach.

\section{Estimation of approximate proportion of Fleas in the Stomach of which multiplication of Plague Bacilli takes place.}

We have now to pass on to consider if any estimation can be made, even approximately, of the proportion of fleas in the stomachs of which multiplication of the plague bacilli takes place. In this connection it is necessary to point out that, of the fleas which are caught on a rat dead of plague, it is always impossible to say how many have imbibed blood containing bacilli, and how many, not having fed at all, or having fed at a time when no plague bacilli were present in the blood, did not take bacilli into their stomachs. If, then, a certain number of fleas, which have been taken from a septicaemic plague rat, be examined day by day and the number containing abundant plague bacilli in their stomachs be noted we can calculate the percentage of those in which multiplication of the bacilli has taken place only in terms of the total number which was exposed to infection and not in terms of those which 
took bacilli into their stomachs. Further, it is obvious that the number of fleas found infected, that is to say, containing abundant plague bacilli in their stomachs, may be influenced by the interval which has elapsed between the time of their feeding on septicaemic blood and the time of examination. And again it may be influenced by the season of the year at which the experiments were carried out.

The observations which were conducted with the object of answering these questions were made in exactly the same manner as those we have just described, that is to say, the stomach contents of fleas, which had been in contact with septicaemic plague rats, were examined day by day after the death of the rat, the fleas in the meantime being fed on healthy animals. In all three series of experiments were made, each at a different season of the year.

(a) The first series was carried out between the months of December and April, the plague season in Bombay. Table I contains the results, and to those we would now refer.

It is seen from this table that for the first four days after the fleas have had an opportunity of feeding on septicaemic blood 43 per cent. of them contain abundant plague bacilli in their stomachs; that from the 5 th to the 12 th day 13 per cent. were still infected; and that, while the stomach contents of all those examined between the 13th and 19th day were free from germs, still on the 20th day abundant bacilli were found in the stomach contents of one out of 15 fleas dissected.

\section{TABIE II.}

Results of examination of fleas as to the presence of abundant plague bacilli in stomach contents, day by duy after their being taken from septicaemic rats, the fleas in the meantime being fed on healthy animals. May 7 th to June 9th.

\begin{tabular}{|c|c|}
\hline \multicolumn{2}{|c|}{$\begin{array}{l}\text { Interval between } \\
\text { ingestion of } \\
\text { septicaemic blood and } \\
\text { examination of flea }\end{array}$} \\
\hline \multicolumn{2}{|c|}{2 days } \\
\hline 3 & , \\
\hline 4 & , \\
\hline 5 & , \\
\hline 6 & 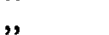 \\
\hline 7 & , \\
\hline 8 & , \\
\hline
\end{tabular}

$\begin{gathered}\text { Number } \\ \text { of fleas } \\ \text { dissected }\end{gathered}$
27
36
36
24
12
21
20

\begin{tabular}{|c|}
\hline $\begin{array}{l}\text { Number of fleas } \\
\text { found with abundant } \\
\text { plague bacilli } \\
\text { in stomach contents }\end{array}$ \\
\hline 0 \\
\hline 5 \\
\hline 0 \\
\hline 2 \\
\hline 0 \\
\hline 3 \\
\hline 0 \\
\hline
\end{tabular}

\begin{tabular}{|c|}
\hline $\begin{array}{l}\text { Percentage of } \\
\text { fleas with abundant } \\
\text { plague bacilli } \\
\text { in stomach content }\end{array}$ \\
\hline 0 \\
\hline 15 \\
\hline 0 \\
\hline 8 \\
\hline 0 \\
\hline 14 \\
\hline 0 \\
\hline
\end{tabular}

(b) The second series was made between May 7th and June 9th, 1906, when epidemic plague had practically died down in Bombay. 
The results of this series are given in Table II, from which it is seen that while some fleas examined contained abundant plague bacilli in their stomachs, this percentage was very much less than in the first series; that only on three out of eight days were infected fleas found; and that none were got after the 7 th day.

(c) The third series of observations was made between 17 th $J$ une and 3rd Sept., 1906, a season when epidemic plague is low, but in which there has been observed in various epidemics in Bombay a slight recrudescence, especially in August.

The results of this series are given in Table III, from which it is seen that only on the 2nd, 3rd, and 5th days were infected fleas found, and that the percentage of infected ones on the total examined was small in comparison with the figures obtained during the plague season.

\section{TABLE III.}

Results of examination of fleas as to the presence of abundant plague bacilli in stomach contents, day by day after their being taken from septicaemic rats, the fleas in the meantime being fed on healthy animals. June 17 th to September $3 r d$.

\begin{tabular}{|c|c|c|c|}
\hline $\begin{array}{c}\text { Interval between } \\
\text { ingestion of } \\
\text { septicaemic blood and } \\
\text { examination of flea }\end{array}$ & $\begin{array}{l}\text { Number } \\
\text { of fleas } \\
\text { dissected }\end{array}$ & $\begin{array}{l}\text { Number of fleas } \\
\text { found with abundant } \\
\text { plague bacilli } \\
\text { in stomach contents }\end{array}$ & $\begin{array}{l}\text { Percentage of } \\
\text { fleas with abundant } \\
\text { plague bacilli } \\
\text { in stomach contents }\end{array}$ \\
\hline 2 deys & 12 & 2 & 16 \\
\hline 3, & 78 & 12 & 16 \\
\hline 4, & 41 & 0 & 0 \\
\hline 5, & 23 & 1 & 4 \\
\hline 6 , & 7 & 0 & 0 \\
\hline
\end{tabular}

In the next table (Table IV) are brought together the results of the examination of all fleas dissected between the 2 nd and 6 th day inclusive. This table shows that during the epidemic plague season the number of fleas found infected between the days mentioned is six times the number found in the non-epidemic season. It is also noteworthy that during

TABLE IV.

Comparison of number of fleas found infected between the 2nd and 6th days after inyestion of septicaemic blood at three seasons of the year.

Season of year

December to April

May 7 th to June 9 th

June 17th to September 3rd

Journ. of Hyg. vII

Number of fleas
found with abundant
plague bacilli
in stomach contents
43
7
15

161

$\begin{gathered}\text { Number of } \\ \text { fleas examined }\end{gathered}$
138
135
161

xamined

$\begin{gathered}\text { Percentage of } \\ \text { fleas with abundant } \\ \text { plague bacilli }\end{gathered}$
in stomach contents
$31 \cdot 2$
$5 \cdot 2$
$9 \cdot 3 \quad$


the season when plague is liable to show a slight recrudescence the number of infected fleas was nearly double the number found in May and first half of June.

\section{Summary.}

1. The percentage of fleas, which have been taken from septicaemic plague rats, found with abundant plague bacilli in their stomach contents varies with the season of the year, being six times greater in the epidemic season than in the non-epidemic season.

2. In the epidemic season this percentage was greatest for the first 4 days, but a certain number was found infected up to the 12th day. On one occasion the stomach contents of a 20 th day flea were found full of plague bacilli.

3. In the non-epidemic season no flea was found with plague bacilli in its stomach after the 7 th day.

\section{Presence of Plague Bacilli in the Rectum and the Faeces of Infected Fleas.}

In the paper dealing with the physiological anatomy of the flea we have seen that the blood after the end of the digestive process in the stomach passes into the rectum as a thick, slimy, dark-red mass and appears at the anus as minute, dark-red or black tarry droplets. We have examined the contents of the rectum for the presence of plague bacilli in the course of the systematic daily dissections of fleas which were made for the observations which have been already described. On very many occasions we have seen the rectal contents crowded with plague bacilli.

As regards the faeces we have examined them both microscopically and by animal tests for the presence of plague bacilli. The following are the results :-

(a) Microscopically.

A number of infected fleas are put into a test-tube; the mouth of the tube is covered over with a glass slide and the tube turned upside down. The fleas are then seen to run about freely over the surface of the slide, and if left for a short time they deposit a considerable amount of faecal matter on this surface. On fixing and staining this smear of faeces it is found that the whole preparation is covered with plague bacilli, their arrangement suggesting that they had been smeared about by the legs of the fleas as they move from place to place. 
(b) Subcutaneous inoculation into Guinea-pigs.

This series of experiments was made by injecting subcutaneously into guinea-pigs an emulsion of the faeces of fleas taken from plagueinfected rats. A number of fleas, between 20 and 30 , which had been taken from rats dead of septicaemic plague, were put into a glass tube, one end of which was covered in with a layer of fine muslin. Through this layer of muslin the fleas were allowed to feed on a healthy guineapig. After they had fed for from 10 to 15 minutes the muslin was always found soiled with faeces. The faeces were then emulsified, and the emulsion injected subcutaneously into guinea-pigs. We have injected 15 guinea-pigs with the faeces of infected fleas collected in this manner. Out of these animals 10 contracted plague. Of these 10 guinea-pigs five died of typical plague between the 3rd and 11th days. The other five, being judged to be plague infected, were killed with chloroform between the 8th and 13th days. In these cases the lesions consisted of swelling and oedema at the site of inoculation; a bubo in the corresponding set of glands; and in two instances coarse granules in the spleen and liver. A culture of the plague bacillus was got from each of these animals.

It is to be noted that these experiments were made during the month of October, 1906, when plague is only sporadic both in rats and man.

(c) Cutaneous inoculation into Guinea-pigs.

We have made a further series of experiments to test the infectivity of the faeces of infected fleas. The faeces were collected on muslin in the same manner as we have described for the preceding experiments. An emulsion made with sterile broth was rubbed into a scarified surface on the abdomen of a guinea-pig. Of nine animals inoculated with this material two died of typical plague.

\section{Summary.}

The rectal contents and faeces of fleas taken from septicaemic plague rats often contain abundant virulent plague bacilli. 


\section{Absence of Plague Bacilli from other Parts of the Body of infected Fleas'.}

We have already seen that it can easily be demonstrated that abundant plague bacilli are present in the stomach and rectum of plague-infected fleas and that multiplication of these bacteria takes place in the stomach. On rare occasions a few bacilli have been seen in the oesophagus, but only in those cases in which the flea has been killed immediately after feeding on septicaemic blood.

As regards other regions of the body we may say at once, that out of more than a hundred infected fleas which have been examined specially for this purpose on no occasion have any plague bacilli been found outside of the organs already mentioned. No infection of the body cavity has been seen, and although particular attention was paid to the salivary glands, nothing at all resembling a plague bacillus has ever been detected in them.

These dissections were carried out on fleas which had come off septicaemic rats at intervals varying from a few hours to several days, the fleas in the meantime being fed on healthy animals.

\section{Duration of Infectivity of Fleas after they have imbibed septicaemic Plague Blood.}

We have already seen that plague bacilli multiply in the stomachs of fleas, and that they can be found there in abundant numbers for at least twelve, and even for twenty days after the insect has imbibed septicaemic blood. It, therefore, was of some importance to test how long fleas taken from plague-infected rats remained infective, that is to say, were capable of transmitting the infection to healthy animals. With this object in view three series of experiments were done.

Series 1. Fleas were infected by placing them in company with Bombay rats, which had received subcutaneous injections of a virulent culture of plague, and in which at death a considerable number of plague bacilli were found in the blood. The fleas were removed from the cage as soon after the death of the rat as possible. They

\footnotetext{
1 In the summary of previous observations on the relation of the plague bacillus to the flea already published (vol. vr. p. 425) we omitted to note that Buchanan (Seventh Annual Report of the Local Government Board for Scotland, 1902, p. 73) found "enormous numbers" of plague bacilli in the "stomach and other parts of the body" of a flea taken from a rat dead of plague in Glasgow.
} 
were then transferred to a clean flea-proof cage ${ }^{1}$ with a small wire cage in the centre, in which a healthy white rat or guinea-pig was placed. The animal was left in the cage for 24 hours. It was then removed, cleaned of fleas, and segregated. The fleas were counted, and returned to the cage, into which a fresh animal was put. This proceeding was repeated each day until no more fleas could be taken on the animals.

Working in this way we have made 19 experiments, the duration of each observation varying from 6 to 15 days. In all 195 animals were exposed to infection and of these animals 29 guinea-pigs developed plague and died.

Table $\mathrm{V}$ gives the details of these observations. From this table it is seen that an average of 97 fleas taken from septicaemic rats were added originally to each cage; that the number of these fleas which could be recovered from the guinea-pigs gradually diminished day by day, until after 15 days an average of only 0.5 per cage was taken.

\section{TABLE V.}

Experiments to ascertain the duration of infectivity of fleas taken from plague rats.

SERIES 1. 10th March to 26th April.

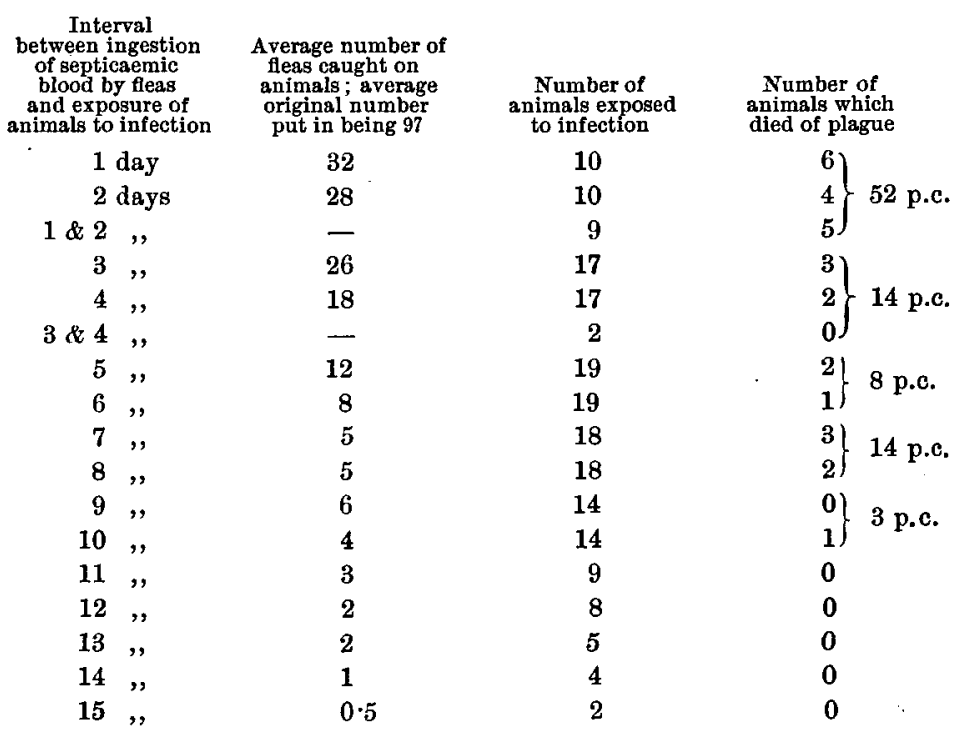

1 The reader is referred to the picture facing page 435 in vol. vI. The present cage was of the same pattern but was a little smaller and had only one wire cage inside. 
Further, it is seen that of the animals exposed to the bites of first and second day fleas, or to fleas of both these days, a little more than half died of plague; that on and after the 3rd day the proportion of animals which became infected fell considerably, but that on each day, except the 9th, up to the 10th some of the animals contracted the disease. Lastly, it is seen that no animal after the 10th day became infected.

Tested in this way we can say that the fleas remained infective for at least ten days, and the probability of their being infective diminished pari passu with the number which could be caught on the animal, and with the length of the interval from the time of feeding the fleas on the infected rat.

It is to be noted that this series of observations was made during the height of the plague epidemic season, 10th March to 26th April.

Series 2. In this series a technique similar to that used in the preceding experiments was employed, the only difference being that, instead of a number of small cages, a single long cage was substituted.

This cage, of the same pattern as that already described, contained inside eight wire cages, which held the experimental animals. It had been used principally as a breeding place for rat fleas, having been started for this purpose on 16th December, 1905, with a stock of 40 fleas. On the 20th April, 1906, when the present experiment was begun, there were several hundred fleas in the cage, all bred from the original forty. On this date six Bombay rats, which had been inoculated on the previous day with a virulent culture of $B$. pestis, were placed separately in the small wire cages. Two of these rats were found dead on the 21st April and the remaining four on the 22nd. The blood of all these rats contained abundant plague bacilli.

On the 22nd April a healthy guinea-pig was put into one of the wire cages and left in for 24 hours. It was then removed, thoroughly cleared of fleas, and segregated. The majority of the fleas were returned to the cage, but a few were dissected and the stomach contents examined microscopically as to the presence or absence of abundant plague bacilli. The same procedure was carried out day by day for 25 days, the only variation being, that from the 10th to the 18th day inclusive, two fresh guinea-pigs instead of one were daily exposed to infection in the cage. From Table VI, which contains the results of these observations, it is seen that up to and including the 8th day a considerable proportion of the fleas examined were found infected; that from the 9 th to the 12 th day although infected 
fleas were still found, their number had diminished, and that from the 13th day onwards only on one occasion, namely, the 20th day, were plague bacilli found in the stomach contents of any of the fleas examined. As regards the fate of the guinea-pigs, it is seen that with one exception, namely, the animal exposed on the 6th day, all guinea-pigs exposed to infection up to and including the 11th day

TABLE VI.

Experiments to ascertain the duration of infectivity of fleas fed on plague-infected rats.

SERIES 2, 20th April to 12th May.

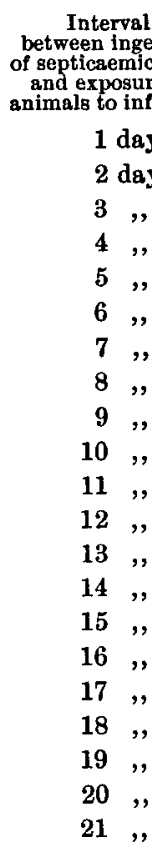

Mean daily temperature $83^{\circ} \mathrm{F}$.

\begin{tabular}{cccc}
$\begin{array}{c}\text { Number } \\
\text { of fleas } \\
\text { examined }\end{array}$ & $\begin{array}{c}\text { Number of fleas } \\
\text { with plague bacilii } \\
\text { in stomach contents }\end{array}$ & $\begin{array}{c}\text { Number of } \\
\text { guines.-pigg } \\
\text { exposed } \\
\text { to infection }\end{array}$ & $\begin{array}{c}\text { Number of } \\
\text { guinea-pigs } \\
\text { which died } \\
\text { of plague }\end{array}$ \\
\hline- & - & 1 & 1 \\
10 & 3 & 1 & 1 \\
15 & 5 & 1 & 1 \\
15 & 8 & 1 & 1 \\
15 & 2 & 1 & 1 \\
13 & 8 & 1 & 0 \\
14 & 2 & 1 & 1 \\
18 & 3 & 1 & 1 \\
13 & 0 & 1 & 1 \\
15 & 1 & 2 & 2 \\
17 & 2 & 2 & 2 \\
22 & 2 & 2 & 0 \\
16 & 0 & 2 & 0 \\
13 & 0 & 2 & 0 \\
6 & 0 & 2 & 1 \\
16 & 0 & 2 & 0 \\
14 & 0 & 2 & 0 \\
14 & 0 & 2 & 0 \\
- & - & 1 & 0 \\
15 & 1 & 1 & 0 \\
19 & 0 & 1 & 0
\end{tabular}

died of plague; and that after this day, only one animal, namely, the one put in on the 15th day, contracted the disease. When we compare day by day the number of fleas which contained bacilli in their stomachs and the number of animals which became infected we find that both diminished equally. The total number of fleas in the cage did not apparently decrease, being kept up by the breeding which was going on. It would appear, therefore, either that the fleas which had originally imbibed bacilli died out as in the previous experiment, 
or that the bacilli disappeared from their stomachs. It is to be noted that this observation was made at the end of April and beginning of May, 1906, when the daily mean temperature was about $83^{\circ} \mathrm{F}$. and when plague, although on the decline, was still epidemic in the city.

Series 3. This experiment was carried out in a similar manner to the last, but was made about five weeks later when the daily mean temperature was about $88^{\circ} \mathrm{F}$, and when the epidemic in Bombay had practically ceased.

The same cage was used as was employed for the last observation. On the 26th May, fleas being very abundant in the cage, eight rats which had been inoculated on the day previous with a virulent culture of Bacillus pestis were put separately into the wire cages. On the 27 th five rats were found dead: in the blood of four of these rats there were abundant plague bacilli, but the blood of the fifth was free from organisms. On the 28th May two of the remaining rats were dead, both with abundant plague bacilli in the blood. The last rat was taken out.

The same procedure was followed as in the previous experiment,

\section{TABLE VII.}

Experiments to ascertain the duration of infectivity of fleas fed on plague-infected rats.

SERIES 3. 20th May to 11th June.

Mean daily temperature $88^{\circ} \mathrm{F}$.

$\begin{array}{ccc}\begin{array}{c}\text { Interval between ingestion } \\ \text { of septicaemic blood } \\ \text { and exposure of } \\ \text { animals to infection }\end{array} & \begin{array}{c}\text { Number of guinea-pigs } \\ \text { exposed to infection }\end{array} & \begin{array}{c}\text { Number of } \\ \text { guinea-pigs which } \\ \text { died of plague }\end{array} \\ 1 \text { day } & 2 & 2 \\ 2 \text { days } & 2 & 0 \\ 3, " & 2 & 1 \\ 4, ", & 2 & 0 \\ 5, ", & 2 & 1 \\ 6, " & 2 & 0 \\ 7,, & 2 & 1 \\ 8, & 2 & 0 \\ 9, & 2 & 0 \\ 10, & 2 & 0 \\ 11, & 2 & 0 \\ 12, & 2 & 0 \\ 13, & 2 & 0 \\ 14, & 2 & 0 \\ 15, & 2 & 0\end{array}$


with the exception that two guinea-pigs were put into the cage each day and all the fleas were returned to the cage, none being kept for dissection.

The result of this experiment is detailed in Table VII, from which it is seen that only on four days, namely, the 1st, 3rd, 5th and 7th day, did either of the animals contract plague, and that only on one occasion, namely, the 1st day, did both the animals before infected.

\section{Summary.}

1. One series of experiments made during the epidemic plague season to test the duration of infectivity of rat fleas fed on septicaemic rats' blood showe? that these fleas could remain infective for at least ten days. This series was made in separate cages with a limited supply of fleas.

2. A second series also made during the epidemic season, but in a single large cage in the presence of a large number of fleas, gave the time that fleas might remain infective as 15 days.

3. In a third series of experiments conducted under the same conditions as the second series but during the non-epidemic season, the fleas remained infective for only seven days, and, further, far fewer (one-third instead of two-thirds) animals than in the second series contracted the disease.

II. Experiments to show that a Single Rat Flea (P. cheopis), taken from a Plague-infected Rat is Rarely able to cause InfECtion of a Healthy ANimal.

In all the experiments on flea transmission which we have carried out, a considerable number of fleas has been used on each occasion.

It was therefore of some interest to ascertain if a single flea taken from a septicaemic plague rat could transmit the infection.

Infected fleas were obtained in the manner we have already described. They were then transferred to healthy white English rats in clean flea-proof cages, one flea being added to each cage.

Sixty-seven experiments were made in this manner, but in only one instance was there a successful transference of the disease. From the result of our dissections of fleas taken from plague-infected rats (vide supra) we can estimate that about half of these 67 fleas would contain plague bacilli in their stomachs. It would appear, therefore, 
that the chances of a non-immune animal, such as the tame white rat, contracting plague after being bitten by a single infected flea are remote.

\section{Experiments to show that both Male and Female Rat Fleas ( $P$. cheopis) can transmit Plague.}

A series of experiments was made to ascertain if both the male and female of $P$. cheopis could transmit plague from animal to animal. Fleas taken from septicaemic plague rats were chloroformed, examined under the low power of the microscope, and separated into male and female. Each lot, of from 15 to 25 individuals, was added to a fresh guinea-pig in a flea-proof cage. Working in this manner we have made six experiments with males and six with females, with the result that one animal in each series became plague infected. The experiment was stopped after this success had been achieved.

\section{Experiments to ascertain if Fleas of Species other than $P$. cheopis can transmit Plague from animal to Animal.}

We have not been able to procure in Bombay any other species of rat flea except $P$. cheopis in numbers sufficient for experimental purposes. Our experiments in this direction have therefore been confined to two species not commonly found on rats, namely $P$. felis, the common $\operatorname{dog}$ and cat flea, and $P$. irritans, the human flea, and to two experiments with Ceratophyllus fasciatus, the common rat flea of Europe, which is found in small numbers on rats in the Punjab.

\section{(a) Experiments with Cat Fleas (P. felis).}

These fleas were for the most part collected from guinea-pigs allowed to run about in places where goats were tethered. The fleas were then placed on Bombay rats which had been inoculated on the previous day with a virulent culture of $B$. pestis. On the death of the rat, if a good septicaemia was present, each flea taken from the cage was placed in a separate test tube. It was then examined by means of a hand lens so as to exclude any rat flea which might be present. The method used for this differentiation is described elsewhere (p. 446). About 20 fleas were then added to a fresh guinea-pig in a clean, flea-proof cage. Working in this way we have carried out 27 experiments with cat fleas without any suc- 
cessful transmission taking place. It may be added that these experiments were made during the height of the plague epidemic in Bombay.

\section{(b) Experiments with Human Fleas (P. irritans).}

The supply of human fleas was got from houses which had been recently evacuated by the inhabitants. A man with bare legs is sent into the house, and remains in for a few minutes. On his coming out his legs are seen to be covered with fleas, which are easily picked off and put into test tubes. The great majority of these fleas belong to the species $P$. irritans, but a few rat fleas can also be taken in this way. The fleas are then infected in the same manner as in the previous experiments. After being infected each flea is examined with a lens to make certain that no rat fleas are introduced. The infected fleas, about 20 for each experiment, are put into a flea-proof cage along with a healthy fresh guinea-pig.

Working in this manner, during the months of April and May, 1906, we have completed 38 experiments with $P$. irritans, with the result that three of the guinea-pigs became plague infected. It is evident, therefore, that while this flea is able to transmit the infection it does not transmit it as readily as Pulex cheopis. An explanation of this difference was obtained when it was found that $P$. irritans does not live well either on rats or on guinea-pigs. A count of the fleas was made daily in a number of experimental cages in which human fleas were living in company with wild Bombay rats. A great number of human fleas, caught in the manner we have already described, were put into a flea-proof cage along with a rat. Each day a census was made of the fleas still alive. After 24 hours it was found that only 1.2 per cent. of the fleas originally put in could be recovered, and after 72 hours this was further reduced to 1 per cent.

Another series of experiments, after the manner we have already described, was begun with the object of ascertaining how long human fleas remain infective, but as no fleas were ever found alive after the 5th day, this series had to be abandoned. However, each day a certain number of fleas were dissected and their stomach contents examined for the presence of plague bacilli. Table VIII contains the results of this examination. From this table it is seen that fleas with large numbers of plague bacilli in their stomach contents were found up to the 4th day, but that the proportion of infected fleas gradually diminished from the 2 nd to the 4 th day. It would appear, however, 
from these results that multiplication of plague bacilli can take place in the stomach of the human flea.

\section{TABLE VIII.}

Results of examination of human fleas ( $P$. irritans) as to the presence of abundant plague bacilli in stomach contents after their being taken from septicaemic rats, the fleas in the meantime being fed on healthy animals.

\begin{tabular}{|c|c|}
\hline \multicolumn{2}{|c|}{$\begin{array}{l}\text { Interval between } \\
\text { ingestion of } \\
\text { septicaemic blood and } \\
\text { examinstion of fleas }\end{array}$} \\
\hline \multicolumn{2}{|c|}{$1 \mathrm{day}$} \\
\hline \multicolumn{2}{|c|}{2 days } \\
\hline 3 & , \\
\hline 4 & ", \\
\hline 5 & \\
\hline
\end{tabular}

Number
of fleas
dissected
2
9
41
14
3

Number of fleas
found with abundant
plague bacilli
in stomach contents
1
5
11
2
0

Percentage of
fleas with abundant
plague bacilli
in stomach contents
50
55
27
14
0

(c) Experiments with Ceratophyllus fasciatus.

As these experiments were made at the end of the observations at Kasel and Dhand, and as this species of flea is obtained in very small numbers, only two complete experiments were carried out. The method used was exactly the same as that already described. The fleas were infected by allowing them to feed on septicaemic plague rats. On the death of the rats they were carefully examined with a lens so as to exclude any $P$. cheopis. They were then transferred to a healthy guinea-pig in a flea-proof cage.

Working in this way two experiments were made, with a successful result in each instance. In one case ten fleas were transferred. The guinea-pig died four days afterwards, and on post-mortem examination showed typical signs of plague, buboes being present in both inguinal regions. Pure cultures of $B$. pestis were obtained from the heart-blood. In the other instance four fleas were transferred. The guinea-pig died 12 days afterwards. Post-mortem examination revealed cervical buboes and other signs of plague. A pure culture of $B$. pestis was obtained from the heart-blood.

These two experiments show definitely that Ceratophyllus fasciatus can convey plague from infected to healthy animals.

Summary.

1. Twenty-seven experiments to transmit plague from animal to animal by means of cat fleas $(P$. felis) were made. None of these was successful. 
2. Thirty-eight experiments to transmit plague from animal to animal by means of human fleas ( $P$. irritans) were made. Three were successful.

3. Two experiments made with C. fasciatus both gave successful results.

\section{Question of the Method by Means of which the Rat Flea (P. cheopis) transmits Plague to a Healthy animal.}

We propose, in conclusion, to consider the question of how the flea transmits its infection to the healthy animal, on which problem some of the observations detailed above have a distinct bearing.

The following methods are possible:-

1. By the animal eating the infected fleas.

2. By the proboscis of the flea mechanically conveying the bacilli from the infected to the healthy animal.

3. By the salivary glands of the flea becoming infected, the bacilli being then inoculated along with the saliva.

4. By a regurgitation of the stomach contents through the oesophagus and pharynx, the bacilli being then injected with the saliva, or on the pricker, or being rubbed into the wounds made by the pricker.

5. By a retention of infected blood in the pharynx or about the mouth parts of the flea, the bacilli multiplying there and then being inoculated into the animal in the same manner as in No. 4 hypothesis.

6. By the bacilli contained in the faeces being deposited on the skin, and then being either injected by the pricker or rubbed into wounds made by the pricker.

Let us consider in detail the data bearing on each of these possible methods.

\section{By the Animal eating the infected Fleas.}

That this means of infection is of any importance, even if it may sometimes occur, is improbatle for the following reasons:

1. Feeding experiments have shown that an animal is unlikely to become infected by the ingestion of material containing plague bacilli, unless the amount is considerable.

2. In 70 per cent. of the cases of infection by feeding, the animals developed a primary mesenteric bubo, but out of several hundred animals infected in the laboratory by meuns of fleas in no case.was a mesenteric bubo discovered. 
3. Infected fleas confined in test tubes readily convey the disease when allowed to bite an animal, in which case the situation of the primary bubo corresponds with the skin area upon which the fleas are placed.

\section{By the Proboscis of the Fleas mechanically conveying the Bacilli from the infected to the healthy animal.}

By this method of infection we mean that the proboscis of the flea acts merely as a mechanical instrument for transference of the bacilli from an infected to a healthy animal. It can be imagined that the external service of the flea's proboscis might become contaminated with plague bacilli while the flea was sucking the blood of a septicaemic rat, and that, on the flea transferring itself to a healthy animal, the proboscis might inject the bacilli under the skin.

It is impossible to exclude this means in the case of animals infected by fleas which have recently left a septicaemic host, but it is significant that the largest number of infections occurred with fleas which had fed upon infected animals within 36 hours. It is difficult, however, to suppose that contamination of the proboscis can explain those cases of continued infectivity for several days during which the insect fed regularly upon healthy animals.

Moreover, microscopical examination of the proboscis of more than 100 infected fleas failed to demonstrate the presence of plague bacilli.

\section{By the salivary glands of the Flea becoming infected, the Bacilli being then inoculated along with the Saliva.}

When it was ascertained that the rat flea could transmit infection from one animal to another, and especially when it was found that the flea might remain infective for several days, the possibility of a salivary gland infection suggested itself. We have carefully searched for the presence of plague bacilli in the body cavity and in the salivary glands of infected fleas. We have dissected and examined hundreds of fleas at different intervals after they had imbibed septicaemic blood, but on no occasion have we been able to find plague bacilli outside of the alimentary canal, and the salivary glands have always been found free from these organisms. 


\section{By a regurgitation of the Stomach Contents through the Oesophagus} and the Pharynx, the Bacilli being then injected with the Saliva or on the Pricker or being rubbed into the wounds made by the Pricker:

We have seen above that the stomach of an infected flea may contain abundant plague bacilli. The hypothesis, which we have now to consider, is that the stomach contents may regurgitate through the pharynx, and that the plague bacilli contained therein may be injected along with the saliva or, soiling the pricker, may be injected by it, or being deposited on the surface of the skin may be rubbed into the pricker wounds.

In the paper on the physiological anatomy of the flea (vol. vi. p. 491) we have noter that the pharyngeal muscles contract like a wave from before backwards, the blood being passed into the stomach as a result of this wave-like action. On one or two occasions by means of a stereoscopic microscope we have been able to see this process taking place. We have no evidence that it may not be reversed, but it seems unlikely that a reverse action should take place.

The anterior end of the stomach is provided with an efficient valvular arrangement with the apparent object of preventing regurgitation, as shown by the following experiment, which has been tried several times. The stomach of a flea which had recently fed was dissected out intact. As long a portion of rectum as possible was left attached posteriorly. The oesophagus having been severed well in front of the valve, pressure was applied with a blunt instrument with the object of extruding the blood through the oesophagus. The posterior passage was closed by the contraction of the rectum. In no instance was it found possible to force the oesophageal passage, although sufficient pressure was applied to rupture the stomach.

On the other hand, in the case of fleas killed by immersion in alcohol, blood has been seen in the pharynx and mouth although the insects had not fed for some hours.

Plague bacilli have only rarely been seen in the oesophagus of plague-infected fleas, and when seen they have been few in number and only in fleas recently taken from plague-infected rats.

A study of the anatomy of the mouth parts of the flea, as described in our previous report, makes it clear that it is improbable that the saliva could become infected by matter regurgitated from the stomach. The regurgitated matter would escape by the mouth and 
would then have to find its way into the salivary grooves in the mandibles. Another possibility is that the regurgitated matter might find its way into the aspiratory canal formed by the epipharynx and the mandibles and pass down that channel. We have no evidence bearing on this point. If regurgitation does take place we have no evidence bearing on the questions of the inoculation of the infected material mechanically by the pricker or its being rubbed in through the pricker wounds.

5. By a retention of infected blood in the Pharynx or about the Mouth Parts of the Flea, the Bacilli multiplying there, and then being inoculated into the Animal in the same manner as in the last case.

A study of the anatomy of the mouth parts of the flea suggests the possibility that plague bacilli, taken in with infected blood, might be retained in the pharynx or in the space between the epipharynx and hypopharynx and, multiplying there, be injected into the healthy animal in one of the methods we have mentioned above, but we have no evidence in favour of this possibility over and above what we have brought forward in the previous section.

We have microscopically examined the pharynx and mouth parts of many fleas taken from septicaemic rats but have never seen any plague bacilli in these situations.

In one or two instances we have seen blood in the pharynx and about the mouth of fleas which had not fed for some hours, and which had been killed by immersion in alcohol.

6. By the Bacilli contained in the Faeces being deposited on the Skin and then being either injected by the Pricker or rubbed into the wounds made by the Pricker.

As mentioned above, plague bacilli after being ingested into the stomach of a rat flea multiply there, and for many days after the flea has been taken from the infected rat, abundant plague bacilli can be demonstrated. We have also drawn attention to the fact that the flea while sucking has a habit of squirting blood per anum; that the faeces of infected fleas contain abundant plague bacilli, which are infective for guinea-pigs both when injected subcutaneously and when inoculated by the cutaneous method.

Experiments were made to ascertain if the pricks made by fleas were of sufficient size to allow plague bacilli to enter the body, no 
other damage to the skin being done. Healthy fleas confined in a test tube were allowed to feed on an area of a guinea-pig's abdomen, the hair on which had been previously cut close without injuring the skin. Immediately afterwards a few drops of septicaemic blood of a rat which had died of plague, or of a virulent culture of $B$. pestis, were lightly spread over this area. The guinea-pig was then segregated. Many successful infections were obtained in this way. Similar experiments were made in which the plague culture was first spread on the skin and afterwards healthy fleas were allowed to feed on the same area. Successful infections were also obtained in this way.

The possibility of infection by the faeces in the manner above described has been demonstrated, but whether this is the usual process we have been unable to ascertain.

\section{General Summary and Conclusions.}

1. The average capacity of a rat flea's stomach is approximately $0.5 \mathrm{c} . \mathrm{mm}$. On this basis a flea imbibing the blood of a plague rat showing a good septicaemia might take as many as 5000 germs into its stomach.

2. Multiplication of the plague bacillus takes place in the stomach of the rat flea.

3. The approximate proportion of fleas in the stomach of which multiplication of plague bacilli takes place has been determined, and it has been shown that this proportion varies with the season of the year, being six times greater in the epidemic season than in the non-epidemic season.

4. Plague bacilli are present in the rectum and faeces of fleas taken from plague rats, and such faeces are infective to guinea-pigs both by cutaneous and by subcutaneous inoculation.

5. On rare occasions plague bacilli have been found in the oesophagus, but never in any other region of the body, such as the body cavity or salivary glands.

6. During the plague season fleas might remain infective for 15 days after imbibing infective blood, but during the non-epidemic season no animal was infective after the 7 th day.

7. A single rat flea may transmit the disease.

8. Both male and female rat fleas can transmit the infection.

9. Experimenting with cat fleas $(P$. felis) and human fleas Journ. of Hyg. viI 
(P. irritans), 27 experiments with the former were unsuccessful, and out of 37 experiments with the latter three successes were obtained. Two experiments were made with $C$. fasciatus: both were successful. Multiplication of the plague bacillus takes place in the stomach of the human flea.

10. The plague bacillus has never been seen in the body cavity or in the salivary glands of infected fleas.

Evidence has been obtained to show that the bite of a healthy flea affords a sufficient avenue for infection by septicaemic blood if it is spread upon the bitten part.

No evidence has been obtained in favour of infection by contaminated mouth parts or regurgitation from the stomach, but the possibility of infection by such means cannot be excluded. 